\title{
AUTOMORPHISMS OF NILPOTENT GROUPS OF CLASS TWO WITH SMALL RANK
}

\author{
THOMAS A. FOURNELLE \\ (Received 3 February 1984) \\ Communicated by $\mathrm{H}$. Lausch
}

\begin{abstract}
Rational abelian groups, that is, torsion-free abelian groups of rank one, are characterized by their types. This paper characterizes rational nilpotent groups of class two, that is, nilpotent groups of class two in which the center and central factor group are direct sums of rational abelian groups. This characterization is done according to the types of the summands of the center and the central factor group. Using these types and some cohomological techniques it is possible to determine the automorphism group of the nilpotent group in question by performing essentially matrix computations.

In particular, the automorphism groups of rational nilpotent groups of class two and rank three are completely described. Specific examples are given of semicomplete and pseudocomplete nilpotent groups.
\end{abstract}

1980 Mathematics subject classification (Amer. Math. Soc.): primary $20 \mathrm{~F} 28$; secondary $20 \mathrm{~F} \mathrm{18,20 \textrm {E }}$ 36.

\section{Introduction}

Torsion-free abelian groups of rank one, that is, subgroups of the rational numbers, are characterized by their types. If $A$ and $B$ are two such groups, then $\operatorname{Hom}(A, B), A \otimes B$, etc. may be described quite conveniently by the types of $A$ and $B$ (Fuchs [2], XIII). In this paper, we are concerned with nilpotent groups $G$ of class two where $Z$, the center of $G$, and $Q=G / Z$ are direct sums of subgroups of the rationals. Using the types of the factors of $Z$ and $Q$ along with the cohomological theory of group extensions we are able to reduce the determination of Aut $G$ to linear algebraic calculations.

(c) 1985 Australian Mathematical Society $0263-6115 / 85 \$ A 2.00+0.00$ 
In Section 5 we make specific calculations for the case in which $A, B$ and $Z$ are subgroups of the rationals and $Q=A \oplus B$. In this case we find that there is an exact sequence

$$
1 \rightarrow \operatorname{Hom}(Q, Z) \rightarrow \text { Aut } G \rightarrow C(\Delta) \rightarrow 1
$$

where $C(\Delta) \leqslant$ Aut $Q$ and $\Delta$ is the cohomology class of the extension $1 \rightarrow Z \rightarrow G$ $\rightarrow Q \rightarrow 1$. We further show that $C(\Delta)$ may take the following forms:

(a) 1 , Aut $A$, Aut $B$, Aut $Q$, or a split extension of the form $\operatorname{Hom}(A, B) \rtimes$ Aut $A$ or $\operatorname{Hom}(A, B) \rtimes$ Aut $B$;

(b) a proper subgroup of Aut $A$ or Aut $B$, or a proper nonabelian subgroup of Aut $Q$.

The groups of the form (a) are describable in terms of the types of $A$ and $B$. The groups of the form (b) are describable in terms of the types of $A$ and $B$ as certain matrix groups.

In addition, we determine $\operatorname{Inn} G$ and hence Aut $G / \operatorname{Inn} G$. In Section 6 we give a sufficient condition for the splitting of the sequence (1.1).

As would be expected the possibilities for Aut $G$ here are somewhat limited (cf. Heineken [3]). However, we are still able to construct some interesting examples. In Section 7, we produce a torsion-free nilpotent group $G$ that is semicomplete, that is, Aut $G=\operatorname{Inn} G$. This group is similar to, but constructed in a completely different manner from the first example of such a group which was provided by Zalesskii [7]. We also construct a group $G$ which is pseudocomplete, that is, Aut $G \cong \operatorname{Inn} G$ but Aut $G / \operatorname{Inn} G \neq 1$.

Section 2 describes the homological methods necessary for the study of Aut $G$. These methods were first developed explicitly, as far as the author knows, by Robinson [4].

\section{Homological methods}

Let $Q$ and $Z$ be abelian groups where $Z$ is regarded as a trivial $Q$-module. Let $\alpha \in$ Aut $Z$ and $\beta \in$ Aut $Q$. Then $\alpha$ and $\beta$ induce $\alpha_{*}, \beta^{*} \in \operatorname{Aut}\left(H^{2}(Q, Z)\right)$. If $Z \mapsto G \rightarrow Q$ is a strictly central extension with cohomology class $\Delta$, then there is an automorphism $\gamma$ of $G$ inducing $\alpha$ and $\beta$ if and only if $\alpha_{*} \Delta=\beta^{*} \Delta$ (Stammbach [5], Proposition II.4.3). Thus, we can study automorphisms of an extension of $Z$ by $Q$ if we have sufficient information about $H^{2}(Q, Z)$. We can gain this information from the Universal Coefficients Theorem which states that there is a natural exact sequence

$$
0 \rightarrow \operatorname{Ext}(Q, Z) \rightarrow H^{2}(Q, Z) \rightarrow \operatorname{Hom}\left(H_{2} Q, Z\right) \rightarrow 0 .
$$


Since $Q$ is abelian we may identify the Schur multiplicator $\mathrm{H}_{2} Q$ with the exterior square $Q \wedge Q$ which may be thought of as a skew symmetric tensor product. Thus, we may replace $\operatorname{Hom}\left(\mathrm{H}_{2} Q, Z\right)$ by $S(Q, Z)$, the group of skew symmetric bilinear forms from $Q \times Q$ to $Z$ and obtain the natural exact sequence

$$
0 \rightarrow \operatorname{Ext}(Q, Z) \rightarrow H^{2}(Q, Z) \stackrel{\theta}{\rightarrow} S(Q, Z) \rightarrow 0
$$

(see Warfield [6], page 29).

The exact nature of the mapping $\theta$ in (2.1) is of importance. If $f: Q \times Q \rightarrow Z$ is a 2-cocycle (or factor set) we define $f^{\prime}(x, y)=f(y, x)$. If $\Delta$ is a cohomology class we choose any 2-cocycle $f \in \Delta$. We let $\theta(\Delta)=f-f^{\prime}$. Note that given $f \in \Delta$ we can construct an extension of $Z$ by $Q$ with cohomology class $\Delta$ as follows: let $G_{f}=\{(z, q) \mid z \in z, q \in Q\}$, and define multiplication by

$$
(z, q)\left(z_{1}, q_{1}\right)=\left(z+z_{1}+f\left(q, q_{1}\right), q+q_{1}\right) .
$$

Elements of $Z$ are, of course, identified with elements of $G_{f}$ by the mapping $z \mapsto(z, 0)$. A simple calculation shows that

$$
\left[(z, q),\left(z_{1}, q_{1}\right)\right]=\left(f\left(q, q_{1}\right)-f^{\prime}\left(q, q_{1}\right), 0\right) .
$$

In nilpotent groups of class two, commutators are skew symmetric and bilinear and hence $f-f^{\prime} \in S(Q, Z)$ (for more details see Warfield [6], Section 5).

The mapping $f \mapsto f^{\prime}$ induces a mapping on the cohomology level $\Delta \mapsto \Delta^{\prime}$. We now define $\Delta_{s}=\Delta+\Delta^{\prime}$. Note that $\Delta_{s} \in \operatorname{Ext}(Q, Z)$. The following result is useful in our later calculations.

Proposition 2.1. Let $\Delta \in H^{2}(Q, Z)$ with $\theta(\Delta)=\psi \in S(Q, Z)$. Let $\alpha \in$ Aut $Z$, $\beta \in$ Aut $Q$. Suppose that $H^{2}(Q, Z)$ has no elements of order 2. Then $\alpha_{*} \Delta=\beta{ }^{*} \Delta$ if and only if $\alpha_{*} \Delta_{s}=\beta^{*} \Delta_{s}$ and $\alpha_{*} \psi=\beta^{*} \psi$.

Proof. Let $f \in \Delta$. Then $\alpha$ and $\beta$ act on $f$ (and thus on $\Delta$ ) as follows:

$$
\left(\alpha_{*} f\right)(x, y)=\alpha(f(x, y)) \quad \text { and } \quad\left(\beta^{*} f\right)(x, y)=f(\beta x, \beta y) .
$$

The action of $\alpha$ and $\beta$ on $\psi$ is determined by their action on the 2-cocycles $f$ and $f^{\prime}$ since $\psi=f-f^{\prime}$. Since $f+f^{\prime} \in \Delta_{s}$, the action of $\alpha$ and $\beta$ on $\Delta_{s}$ is similarly determined.

Necessity being clear we turn to sufficiency and assume that $\alpha_{*} \psi=\beta^{*} \psi$ and $\alpha_{*} \Delta_{s}=\beta^{*} \Delta_{s}$. Since $\psi \in \Delta-\Delta^{\prime}$ and $\Delta_{s}=\Delta+\Delta^{\prime}$ we have on the cohomology level

$$
\alpha_{*} \Delta+\alpha_{*} \Delta^{\prime}=\beta^{*} \Delta+\beta^{*} \Delta^{\prime}, \quad \alpha_{*} \Delta-\alpha_{*} \Delta^{\prime}=\beta^{*} \Delta-\beta^{*} \Delta^{\prime} .
$$

Adding these two equations we obtain $2 \alpha_{*} \Delta=2 \beta^{*} \Delta$. Since $H^{2}(Q, Z)$ has no elements of order 2 , the result now follows. 


\section{3. $S(Q, Z), \operatorname{Hom}(A, B)$ and Aut $Q$}

We now restrict our attention to the case in which $A, B$ and $Z$ are non-trivial subgroups of the rationals and where $Q=A \oplus B$.

Since homomorphisms between subgroups of the rationals are induced by multiplication by a fixed rational number it follows easily that if $\psi \in S(Q, Z)$, then $\psi((a, b),(c, d))=k(a d-b c)$ for some fixed $k$ (actually, $k=$ $\psi((1,0),(0,1)))$. Hence $S(Q, Z)$ is isomorphic to some subgroup of the rationals and is torsion-free. Since we are considering extensions $\Delta: Z \gg G \rightarrow Q$ in which $Z$ is the center of $G$ it follows that if $\theta(\Delta)=\psi$ then $\psi((a, b),(c, d))=k(a d-b c)$ for some non-zero $k$. This induces injections of $A$ and $B$ into $Z$. Upon multiplication by an appropriate rational number we may assume without loss of generality that $A, B$ and $Z$ all contain 1 .

Now let $p_{1}, p_{2}, \ldots$ be the sequence of prime numbers in their usual order. We let $\chi_{a}=\left(h_{1}, h_{2}, \ldots\right)$ be the characteristic of 1 in $A$, that is, $h_{i}$ is the $p_{i}$-height of 1 in $A$. Hence $h_{i}$ is either a non-negative integer or $\infty$. Similarly we let $\chi_{B}=$ $\left(k_{1}, k_{2}, \ldots\right)$ be the characteristic of 1 in $B$. Two characteristics are equivalent if they have the same $\infty$ entries and they differ in only finitely many noninfinite coordinates. Equivalence classes of characteristics are types. We let $\tau(A)$ and $\tau(B)$ denote the types of $A$ and $B$, respectively. Subgroups of the rationals are completely characterized by their types.

The product of characteristics is defined by componentwise addition. This induces a product of types. We may similarly define $\tau(A) \leqslant \tau(B)$ componentwise. If $\tau(A) \leqslant \tau(B)$ we define the quotient $\tau(B): \tau(A)$ to be the largest type $\tau$ such that $\tau \tau(A)=\tau(B)$. The proof of the following may be found in Fuchs [2, Vol. 2, page 111].

LEMMA 3.1. If $\tau(A) \leqslant \tau(B)$, then $\operatorname{Hom}(A, B)$ is isomorphic to a subgroup of the rationals of type $\tau(B): \tau(A)$. Otherwise $\operatorname{Hom}(A, B)=0$.

We now wish to determine $\operatorname{Aut}(A \oplus B)$. The homomorphisms between subgroups of the rationals are induced by multiplication by some rational number. Thus if we denote elements of $A \oplus B$ by column vectors then we may associate automorphisms of $A \oplus B$ with rational matrices as follows:

$$
\left(\begin{array}{ll}
r & s \\
t & u
\end{array}\right)\left(\begin{array}{l}
a \\
b
\end{array}\right)=\left(\begin{array}{l}
r a+s b \\
t a+u b
\end{array}\right)
$$

Here $r \in$ Aut $A, u \in$ Aut $B, s \in \operatorname{Hom}(B, A)$ and $t \in \operatorname{Hom}(A, B)$. Thus, $\operatorname{Aut}(A$ $\oplus B)$ is a group of matrics. We have essentially three cases. 
First suppose that $A$ and $B$ have incomparable types. Then $\operatorname{Hom}(A, B)=0=$ $\operatorname{Hom}(B, A)$ and $\operatorname{Aut}(A \oplus B)=($ Aut $A) \oplus \operatorname{Aut}(B)$.

Next, suppose $\tau(A)<\tau(B)$. Then $\operatorname{Hom}(B, A)=0$ but $\operatorname{Hom}(A, B) \neq 0$. Thus

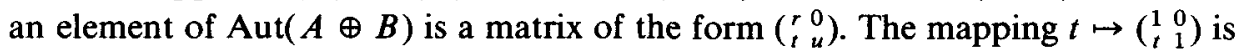
an injection $\operatorname{Hom}(A, B) \nrightarrow \operatorname{Aut}(A \oplus B)$ and from this we see that $\operatorname{Aut}(A \oplus B)$ is is a split extension: $\operatorname{Aut}(A \oplus B)=\operatorname{Hom}(A, B) \rtimes($ Aut $A \oplus$ Aut $B$ ).

Finally, suppose that $A$ and $B$ have the same type. Then we may assume $A=B$ and obtain $\operatorname{Aut}(A \oplus A)=G L(2$, End $A)$ where End $A$ is the endomorphism ring of $A$. Now multiplication by $1 / p$ induces an endomorphism of $A$ exactly when 1 is infinitely divisible by $p$ in $A$. Thus, End $A$ is a subring of the rational numbers in which, when considered as an additive group, the characteristic of 1 has only 0 and $\infty$ entries, and the $\infty$ entries occur precisely in those coordinates for which $\chi_{A}$ has $\infty$ entries. Of course, Aut $A$ is merely the group of multiplicative units in End $A$.

\section{4. $\operatorname{Ext}(Q, Z)$}

In order to compute $H^{2}(Q, Z)$ we can use the Universal Coefficients Theorem. We thus need to know $\operatorname{Ext}(Q, Z)$. Since $Q=A \oplus B$ and $\operatorname{Ext}(A \oplus B, Z)=$ $\operatorname{Ext}(A, Z) \oplus \operatorname{Ext}(B, Z)$ we will only compute $\operatorname{Ext}(A, Z)$.

To this end we let $\chi_{A}=(h(1), h(2), \ldots)$ and $\chi_{Z}=(k(1), k(2), \ldots)$. By our previous remarks we may assume that $h(n) \leqslant k(n)$ for all $n$. We have a short exact sequence

$$
0 \rightarrow \mathbf{Z} \stackrel{\leftrightarrow}{\rightarrow} A \rightarrow A / \mathbf{Z} \rightarrow 0 .
$$

This gives rise to a long exact sequence $[2,51.3]$ which in our case may be shortened to

$$
\text { (3.1) } 0 \rightarrow \operatorname{Hom}(A, Z) \rightarrow \operatorname{Hom}(\mathbf{Z}, Z) \rightarrow \operatorname{Ext}(A / \mathbf{Z}, Z) \rightarrow \operatorname{Ext}(A, Z) \rightarrow 0 \text {. }
$$

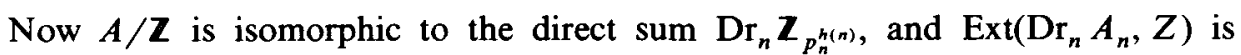
isomorphic to the Cartesian (unrestricted) $\operatorname{sum} \operatorname{Cr}_{n} \operatorname{Ext}\left(A_{n}, Z\right)$. Thus, in (3.1) we

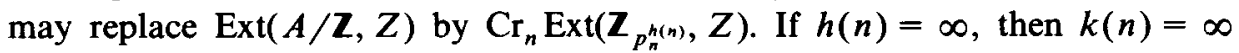
and $Z$ is divisible by $p_{n}$. Since $Z_{p_{n}^{\infty}}$ is a $p_{n}$-group it follows that $\operatorname{Ext}\left(Z_{p_{n}^{\infty}}, Z\right)=0$ [2, Vol. 1, p. 223, $K]$. If $h(n)<\infty$, then $\operatorname{Ext}\left(Z_{p_{n}^{h(n)}}, Z\right) \cong Z / p_{n}^{h(n)} Z[2$, Vol. 1, p. $222, D]$. If $Z$ is $p$-divisible, then $Z / p^{n} Z=0$. If $Z$ is not $p$-divisible, then $Z / p^{n} Z \cong \mathbf{Z}_{p^{n}}$. Hence, if we let $T=\{n \mid h(n) \neq 0, k(n)<\infty\}$ then we have an exact sequence

$$
0 \rightarrow \operatorname{Hom}(A, Z) \stackrel{\iota^{*}}{\rightarrow} \operatorname{Hom}(\mathbf{Z}, Z) \stackrel{\delta}{\rightarrow} \underset{n \in T}{\operatorname{Cr}} \mathbf{Z}_{p_{n}^{n(n)}} \rightarrow \operatorname{Ext}(A, Z) \rightarrow 0 .
$$


Now let $C=\operatorname{Hom}(\mathbf{Z}, Z) / \iota^{*}(\operatorname{Hom}(A, Z))$. If $\theta \in \operatorname{Hom}(\mathbf{Z}, Z)$ then $\theta(x)=(r / s) x$ for some rational number $r / s$. Thus, $s \theta(x)=r x$ and $s \theta$ extends to a homomorphism $\eta \in \operatorname{Hom}(A, Z)$ and $\iota^{*} \eta=s \theta$. Thus, $\theta$ has order $s$ in $C$, and $C$ is a torsion group. We thus have an exact sequence.

$$
0 \rightarrow C \stackrel{\delta}{\rightarrow} \underset{n \in T}{\mathrm{Cr}_{T}} \mathbf{Z}_{p_{n}^{h(n)}} \rightarrow \operatorname{Ext}(A, Z) \rightarrow 0 .
$$

Also, $C \cong \operatorname{Dr}_{n \in T} \mathbb{Z}_{p_{n}^{h(n)}}$ is the torsion subgroup of $\mathrm{Cr}_{n \in T} \mathbb{Z}_{p_{n}^{h(n)}}$. We have proved the following result.

Proposition 4.1. Let $1 \in A \leqslant Z$ be subgroups of the rationals. Then

$$
\operatorname{Ext}(A, Z) \simeq\left(\underset{n \in T}{\operatorname{Cr} \mathbf{Z}_{p_{n}^{h(n)}}}\right) /\left(\underset{n \in T}{\left.\operatorname{Dr} \mathbf{Z}_{p_{n}^{h(n)}}\right)} .\right.
$$

REMARK. For the sake of completeness we mention that if $k(n)=k<\infty$ and $h(n)=\infty$, then $\operatorname{Ext}\left(\mathbf{Z}_{p_{n}^{\infty}}, Z\right)$ is an extension of $\operatorname{End}\left(\mathbf{Z}_{p_{n}^{\infty}}\right)$ by $\mathbf{Z}_{p_{n}^{k}}$.

As a consequence of 3.1 we have that $\operatorname{Ext}(A, Z)=0$ if $T$ is finite. If $T$ is infinite then $\operatorname{Ext}(A, Z)$ is uncountable, torsion-free, and divisible. Now multiplication in $A$ or $Z$ by an integer $n$ induces multiplication in $\operatorname{Ext}(A, Z)$ by $n[2$, 52.1]. Since $\operatorname{Ext}(A, Z)$ is torsion-free and divisible it follows that multiplication in $A$ or $Z$ by some rational number induces multiplication $\operatorname{in} \operatorname{Ext}(A, Z)$ by that same rational number.

LEMMA 4.2. Suppose that $A, B, Z$ are subgroups of the rationals and $\chi_{A} \chi_{B} \leqslant \chi_{Z}$. Let $\iota: A \rightarrow B$ be the injection map. Then $\iota^{*}: \operatorname{Ext}(B, Z) \rightarrow \operatorname{Ext}(A, Z)$ is always an epimorphism which may have a non-trivial kernel.

Proof. From $0 \rightarrow A \stackrel{\iota}{\rightarrow} B \rightarrow B / A \rightarrow 0$ we obtain the long exact sequence

$$
\begin{aligned}
0 & \rightarrow \operatorname{Hom}(B, Z) \stackrel{\iota^{*}}{\rightarrow} \operatorname{Hom}(A, Z) \stackrel{\delta}{\rightarrow} \operatorname{Ext}(B / A, Z) \\
& \rightarrow \operatorname{Ext}(B, Z) \stackrel{\iota^{*}}{\rightarrow} \operatorname{Ext}(A, Z) \rightarrow 0 .
\end{aligned}
$$

Thus $\iota^{*}: \operatorname{Ext}(B, Z) \rightarrow \operatorname{Ext}(A, Z)$ is certainly an epimorphism. Let $\chi_{A}=$ $(h(1), h(2), \ldots), \chi_{B}=(k(1), k(2), \ldots)$ and $\chi_{Z}=(i(1), i(2), \ldots)$. Define $T$ to be $\{n \mid i(n)<\infty$ and $k(n) \neq 0\}$. Then $\operatorname{Ext}(B, Z) \cong\left(\operatorname{Dr}_{n \in T} \mathbb{Z}_{\left.p_{n}^{k(n)}\right)} /\left(\mathrm{Cr}_{n \in T} \mathbf{Z}_{p_{n}^{k(n)}}\right)\right.$. If $\operatorname{Ext}(B, Z) \neq 0$ it follows that $T$ is infinite.

Now $B / A=\operatorname{Dr}_{n} Z_{p_{n}^{k(n) h(n)}}($ where $\infty-\infty=0$ and $\infty-m=\infty$ for finite $m$ ).

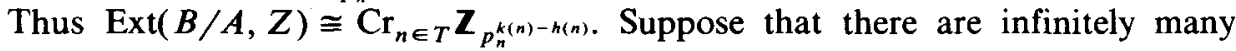
$n \in T$ such that $k(n)>h(n)$. Then $\operatorname{Ext}(B / A, Z)$ is uncountable. Now Ker $\iota^{*} \cong$ $\operatorname{Ext}(B / A, Z) / \operatorname{Im} \delta$, but $\operatorname{Im} \delta$ is countable and thus $\operatorname{Ker} \iota^{*}$ is uncountable and this completes the proof. 
We now wish to describe the action of Aut $Q$ on $\operatorname{Ext}(Q, Z)=\operatorname{Ext}(A, Z) \oplus$ $\operatorname{Ext}(B, Z)$. Let $\beta \in$ Aut $Q$ and $\left(\sigma_{1}, \sigma_{2}\right) \in \operatorname{Ext}(A, Z) \oplus \operatorname{Ext}(B, Z)$. We have essentially three cases to consider.

Case $1 . A$ and $B$ are of incomparable type.

Then Aut $Q=$ Aut $A \oplus$ Aut $B$ and $\beta(a, b)=(r a, s b)$ for some rational numbers $r$ and $s$. Then $\beta^{*}\left(\sigma_{1}, \sigma_{2}\right)=\left(r \sigma_{1}, s \sigma_{2}\right)$.

Case 2. $\tau(A)<\tau(B)$.

Then we have an injection $0 \rightarrow A \stackrel{\iota}{\rightarrow} B$, and $\beta$ may be represented by the matrix $\left(\begin{array}{c}r \\ s_{1}\end{array}\right)$. It follows that $\beta^{*}$ may also be represented by matrix multiplication:

$$
\beta^{*}\left(\sigma_{1}, \sigma_{2}\right)=\left(\sigma_{1}, \sigma_{2}\right)\left(\begin{array}{ll}
r & 0 \\
s \iota^{*} & t
\end{array}\right)=\left(r \sigma_{1}+s \iota^{*} \sigma_{2}, t \sigma_{2}\right) .
$$

Case 3. $A=B$.

The identity map on $A$ induces the indentity on $\operatorname{Ext}(A, Z)$. If $\beta$ is represented by $\left(\begin{array}{cc}r s \\ 1\end{array}\right)$ then it follows that

$$
\beta^{*}\left(\sigma_{1}, \sigma_{2}\right)=\left(\sigma_{1}, \sigma_{2}\right)\left(\begin{array}{ll}
r & s \\
t & u
\end{array}\right)=\left(r \sigma_{1}+t \sigma_{2}, s \sigma_{1}+u \sigma_{2}\right) .
$$

\section{Aut $G$}

Let $Z \leadsto G \rightarrow Q$ be a strictly central extension with cohomology class $\Delta$ where $Q=A \oplus B$ and $A, B$ and $Z$ are subgroups of the rationals (each assumed without loss of generality to contain 1). Since $\operatorname{Ext}(Q, Z)$ and $S(Q, Z)$ are torsion-free, the Universal Coefficients Theorem in the form of (2.1) implies that $H^{2}(Q, Z)$ is torsion-free. Hence, Proposition 2.1 applies.

Now let $\gamma \in$ Aut $G$. Since $Z$ is characteristic in $G$ it follows that $\gamma$ induces $\alpha \in$ Aut $Z$ and $\beta \in$ Aut $Q$. Hence $\alpha_{*} \Delta_{s}=\beta^{*} \Delta_{s}$ and $\alpha_{*} \psi=\beta^{*} \psi$ where $\theta(\Delta)=\psi$ $\in S(Q, Z)$ and $\theta$ is the map in (2.1). Consequently,

$$
\begin{aligned}
\left(\beta^{*} \psi\right)((a, b),(c, d)) & =\psi(\beta(a, b), \beta(c, d))=(\operatorname{Det} \beta) k(a d-b c) \\
& =\left(\alpha_{*} \psi\right)((a, b),(c, d))=\alpha(k(a d-b c)) .
\end{aligned}
$$

Thus $\beta^{*} \psi=\alpha_{*} \psi$ if and only if $\alpha$ is multiplication by Det $\beta$.

We now define $C(\Delta)$ to be the set of all $\beta \in$ Aut $Q$ such that $\beta^{*} \Delta_{s}=(\operatorname{Det} \beta) \Delta_{s}$.

Proposition 5.1. There is an exact sequence

$$
1 \rightarrow \operatorname{Hom}(Q, Z) \rightarrow \text { Aut } G \rightarrow C(\Delta) \rightarrow 1 .
$$

Proof. We let $H$ denote the subgroup of Aut $G$ consisting of those automorphisms which induce the identity in $Z$ and $Q$. If $\gamma \in H$ and $g \in G$, then 
$\gamma(g)=g z_{g}$ for some $z_{g} \in Z$. The mapping $g \rightarrow z_{g}$ is a homomorphism $G \rightarrow Z$ whose kernel includes $Z$. Hence there is an induced homomorphism $\theta_{\gamma}: Q \rightarrow Z$. The mapping $\gamma \mapsto \theta_{\gamma}$ is an isomorphism between $H$ and $\operatorname{Hom}(Q, Z)$. Therefore we have an injection $1 \rightarrow \operatorname{Hom}(Q, Z) \rightarrow$ Aut $G$.

Now let Aut $G \rightarrow$ Aut $Q$ by $\gamma \mapsto \beta$ where $\beta$ is the automorphism of $Q$ induced by $\gamma$. If $\gamma$ also induces $\alpha$ on $Z$, then $\alpha$ is multiplication by Det $\beta$. Hence $\beta \in C(\Delta)$ and we have an epimorphism Aut $G \rightarrow C(\Delta) \rightarrow 1$. The kernel of this epimorphism consists of those $\gamma$ inducing the indenity on $Q$ and hence also on $Z$, that is, the kernel may be identified with $\operatorname{Hom}(Q, Z)$ and the proof is complete.

Now an inner automorphism of $G$ induces the identity on $Q$ and $Z$ and hence Inn $G$ may be identified with a subgroup of $\operatorname{Hom}(Q, Z)$. Let $\operatorname{Hom}\left(A_{B}, Z\right)$ by those homomorphisms from $A$ to $Z$ induced by multiplication by elements of $B$ and define $\operatorname{Hom}\left(B_{A}, Z\right)$ similarly. Also suppose $\psi((a, b),(c, d))=k(a d-b c)$. Then we have the following.

LEMMA 5.2. Inn $G$ may be identified with $k \operatorname{Hom}\left(A_{B}, Z\right) \oplus k \operatorname{Hom}\left(B_{A}, Z\right)$.

Proof. Let $\gamma$ be the inner automorphism of $G$ induced by $x$. Then $\gamma(g)=$ $x^{-1} g x=g[g, x]=g \psi(\bar{g}, \bar{x})$ where $\bar{g}$ and $\bar{x}$ are the images of $g$ and $x$ in $Q$. Thus $\gamma$ is associated with the homomorphism in $\operatorname{Hom}(Q, Z)$ given by $\theta(\bar{g})=\psi(\bar{g}, \bar{x})$. Now let $\bar{g}=(a, b)$ and $\bar{x}=(c, d)$. Then $\theta(a, b)=\psi((a, b),(c, d))=k(a d-$ $b c)=(k d) a+(k(-c)) b$. The result now follows.

The rest of this section is an explication of the various possibilities for $C(\Delta)$. This is done by examining $\beta^{*} \Delta_{s}$. Note that if $\Delta_{1}$ is a preimage of $\psi$ under $\theta$ in (2.1) then we may let $\Delta=\Delta_{1}+\sigma$ where $\sigma \in \operatorname{Ext}(Q, Z)$. Then $\Delta$ is still a preimage of $\psi$. Now $\Delta_{s}=\left(\Delta_{1}\right)_{s}+2 \sigma$. Since $\operatorname{Ext}(Q, Z)$ is divisible, $2 \operatorname{Ext}(Q, Z)=$ $\operatorname{Ext}(Q, Z)$. Thus, if $\Delta$ is a preimage $\psi$ then $\Delta_{s}$ is a completely arbitrary element of $\operatorname{Ext}(Q, Z)$.

We now consider the possibilities for $C(\Delta)$. First note that if $\Delta_{s}=0$ then $C(\Delta)=$ Aut $Q$. Now let $\beta \in$ Aut $Q$. We need to determine whether or not

$$
\beta^{*} \Delta_{s}=(\operatorname{Det} \beta) \Delta_{s} \text {. }
$$

Case 1. $A$ and $B$ are of incomparable types.

Let $\beta \in$ Aut $Q$. Then $\beta(a, b)=(r a, s b)$ for some rational numbers $r$ and $s$.

(i) Let $\Delta_{s}=\left(\sigma_{1}, \sigma_{2}\right)$ where $\sigma_{1}$ and $\sigma_{2}$ have infinite order. Then (5.1) holds if and only if $r s\left(\sigma_{1}, \sigma_{2}\right)=\left(r \sigma_{1}, s \sigma_{2}\right)$, that is, if and only if $r s=r$ and $r s=s$, and hence if and only if $r=s=1$. Hence $C(\Delta)=1$.

(ii) Let $\Delta_{s}=\left(\sigma_{1}, 0\right)$ where $\sigma_{1}$ has infinite order. Then (5.1) holds if and only if $s=1$. Thus, $C(\Delta)=$ Aut $A$.

(iii) Let $\Delta_{s}=\left(0, \sigma_{2}\right)$ where $\sigma_{2}$ is of infinite order. Then (5.1) holds if and only if $r=1$. Thus $C(\Delta)=$ Aut $B$. 
Case 2. $\tau(A)<\tau(B)$.

Then we have an injection $\iota: A \rightarrow B$ and $\beta^{*}$ acts according to right multiplication by the matrix $\left(\begin{array}{cc}r & 0 \\ s i & t\end{array}\right)$.

(i) Let $\Delta_{s}=\left(\sigma_{1}, \sigma_{2}\right)$ where $\sigma_{1}$ and $\sigma_{2}$ have infinite order. Then (5.1) holds if and only if $\left(r t \sigma_{1}, r t \sigma_{2}\right)=\left(r \sigma_{1}+s i * \sigma_{2}, t \sigma_{2}\right)$, if and only if $(r t-r) \sigma_{1}=s i * \sigma_{2}$ and $r t=t$, and thus if and only if $r=1$ and $(t-1) \sigma_{1}=s \iota * \sigma_{2}$.

Suppose first that $\sigma_{1}$ and $\iota * \sigma_{2}$ are linearly independent over the rationals. Then (5.1) holds if and only if $r=1, t=1$, and $s=0$. Thus $C(\Delta)=1$.

Next, suppose that $\iota^{*} \sigma_{2}=0$, which is certainly possible. Then (5.1) holds if and only if $r=t=1$ and $s$ is arbitrary. Thus, $C(\Delta)=\operatorname{Hom}(A, B)$.

Finally, suppose there are non-zero integers $m$ and $n$ such that $m \sigma_{1}=n \iota^{*} \sigma_{2}$. Then (5.1) holds if and only if $s=(m / n)(t-1)$. Thus $C(\Delta)$ consists of matrices of the form

$$
\beta=\left(\begin{array}{cc}
1 & 0 \\
(m / n)(t-1) & t
\end{array}\right)
$$

The mapping $\beta \mapsto t$ is a monomorphism from $C(\Delta)$ to Aut $B$. Thus $C(\Delta) \leqslant$ Aut $B$.

(ii) Let $\Delta_{s}=\left(\sigma_{1}, 0\right)$ where $\sigma_{1}$ has infinite order. Then (5.1) holds if and only if $r=r t$, that is, if and only if $t=1$. Thus $C(\Delta)$ consists of those matrices of the form $\left(\begin{array}{ll}r & 0 \\ s & 1\end{array}\right)$. Hence $C(\Delta)$ is a split extension $\operatorname{Hom}(A, B) \rtimes$ Aut $A$.

(iii) Let $\Delta_{s}=\left(0, \sigma_{2}\right)$ where $\sigma_{2}$ has infinite order. Then (5.1) holds if and only if $s \iota * \sigma_{2}=0$ and $r t=t$.

Now if $\iota * \sigma_{2} \neq 0$, then (5.1) holds if and only if $s=0$ and $r=1$. Hence, $C(\Delta)=$ Aut $B$.

If $\psi^{*} \sigma_{2}=0$, then $C(\Delta)$ consists of matrices of the form $\left(\begin{array}{ll}1 & 0 \\ s & t\end{array}\right)$, and $C(\Delta)=$ $\operatorname{Hom}(A, B) \rtimes$ Aut $B$.

Case 3. $A=B$.

Then $\beta^{*}$ acts according to right matrix multiplication by the matrix $\left(\begin{array}{ll}r & s \\ 1 & u\end{array}\right)$.

(i) Suppose $\Delta_{s}=\left(\sigma_{1}, 0\right)$ where $\sigma_{1}$ is of infinite order. Then (5.1) holds if and only if $r=r u-s t$ and $s=0$, if and only if $s=0$ and $u=1$. Hence, $C(\Delta)=$ $\operatorname{Hom}(A, B) \rtimes$ Aut $A$.

(ii) The situation in which $\Delta_{s}=\left(0, \sigma_{2}\right), \sigma_{2} \neq 0$ is symmetric to the previous case and thus $C(\Delta)=\operatorname{Hom}(B, A) \rtimes$ Aut $B$.

(iii) Suppose $\Delta_{s}=\left(\sigma_{1}, \sigma_{2}\right)$ where $\sigma_{1}$ and $\sigma_{2}$ both have infinite order. Then (5.1) holds if and only if $(r u-s t) \sigma_{1}=r \sigma_{1}+t \sigma_{2}$ and $(r u-s t) \sigma_{2}=s \sigma_{1}+u \sigma_{2}$.

Suppose that $\sigma_{1}$ and $\sigma_{2}$ are linearly independent over the rationals. Then (5.1) holds if and only if $t=0, s=0, r=1$, and $u=1$. Hence $C(\Delta)=1$. 
On the other hand, suppose there are non-zero integers $m$ and $n$ such that $m \sigma_{1}=n \sigma_{2}$. Then (5.1) holds if and only if $n(r u-s t-r)=t m$ and $m(r u-s t-$ $u)=s n$. While these conditions are a bit more complicated than the previous ones, it can easily be seen that they are satisfied by the matrices $\left(\begin{array}{cc}1 & 0 \\ m & m+1\end{array}\right)$ and $\left(\begin{array}{cc}n+1 & m \\ 0 & 1\end{array}\right)$. Since these matrices do not commute it follows that $C(\Delta)$ is a non-abelian subgroup of Aut $Q$.

\section{Splitting Aut $G$}

Suppose that $\Delta_{1} \in H^{2}(Q, Z)$ and that $\Delta_{1} \notin \operatorname{Ext}(Q, Z)$. Let $\Delta=\Delta_{1}-\Delta_{1}^{\prime}$. If $g$ is a 2-cocycle with cohomology class $\Delta_{1}$, then $f=g-g^{\prime}$ is a 2-cocycle with class $\Delta$. Now $\Delta_{s}=0$ and thus if $Z \mapsto G \rightarrow Q$ is an extension with class $\Delta$ then we have an exact sequence

$$
1 \rightarrow \operatorname{Hom}(Q, Z) \rightarrow \text { Aut } G \rightarrow \text { Aut } Q \rightarrow 1 .
$$

Notice that $f$ is actually a skew symmetric bilinear form and hence $f((a, b),(c, d))=k(a d-b c)$. We may realize $G$ as the set of ordered pairs $(z,(a, b)), z \in Z,(a, b) \in A \oplus B=Q$ with operation

$$
(z,(a, b))(w,(c, d))=(z+w+k(a d-b c),(a+c, b+d)) .
$$

Now let $\beta \in$ Aut $Q$. We define $\gamma$ on $G$ as follows:

$$
\gamma(z,(a, b))=((\operatorname{Det} \beta) z, \beta(a, b)) .
$$

An easy calculation now shows that $\gamma \in$ Aut $G$ and the set of all $\gamma$ of the form (6.2) forms a subgroup of Aut $G$ that is mapped onto Aut $Q$ in (6.1). Thus, the extension (6.1) splits.

\section{Specific examples}

Let $\pi$ and $\rho$ be infinite disjoint sets of primes. Let $A, B$ and $Z$ be additive subgroups of the rationals defined as follows:

$$
A=\left\langle\frac{1}{p} \mid P \in \pi\right\rangle ; \quad B=\left\langle\frac{1}{p} \mid p \in \rho\right\rangle ; \quad Z=\left\langle\frac{1}{p} \mid p \in \pi \cup \rho\right\rangle .
$$

Let $Q=A \oplus B$. Then $\operatorname{Ext}(A, Z)$ and $\operatorname{Ext}(B, Z)$ are uncountable. We let $\psi \epsilon$ $S(Q, Z)$ be defined by $\psi((a, b),(c, d))=k(a d-b c)$ where $k$ is a natural number not divisible by any prime in $\pi \cup \rho$. Then we choose $\Delta \in H^{2}(Q, Z)$ to be a preimage of $\psi$ such that $\Delta_{s}=\left(\sigma_{1}, \sigma_{2}\right) \in \operatorname{Ext}(A, Z) \oplus \operatorname{Ext}(B, Z)$ where $\sigma_{1}$ and 
$\sigma_{2}$ have infinite order. Now let $Z \nrightarrow G \rightarrow Q$ be an extension with cohomology class $\Delta$. Since $A$ and $B$ have incomparable types it follows that $C(\Delta)=1$ and

$$
\text { Aut } \begin{aligned}
G & \cong \operatorname{Hom}(Q, Z) \cong \operatorname{Hom}\left(A_{B}, Z\right) \oplus \operatorname{Hom}\left(B_{A}, Z\right) \\
& \cong \operatorname{Hom}(A, Z) \oplus \operatorname{Hom}(B, Z) \cong B \oplus A .
\end{aligned}
$$

If $k=1$, then Aut $G=\operatorname{Inn} G$ and $G$ is semicomplete. If $k>1$, then Aut $G \cong \operatorname{Inn} G$ and Aut $G / \operatorname{Inn} G \cong \mathbf{Z}_{k} \oplus \mathbf{Z}_{k}$ and $G$ is pseudocomplete (see also [1]).

Remark. From certain points of view it might be appropriate to describe the above examples in some more "concrete" fashion, for example, in terms of generators and realtors. While the translation from the cohomological description of the groups given here to a generator-relator description is certainly possible, the method of translation is a bit lengthy to include here. Indeed, the utility of the cohomological methods used here for describing Aut $G$ lies in the fact that they avoid lengthy computations with generators and relators.

\section{References}

[1] T. A. Fournelle, 'Pseudocomplete nilpotent groups', Proc. Amer. Math. Soc. 88 (1983), 1-7.

[2] L. Fuchs, Infinite Abelian groups, (Academic Press, New York and London, 1970, 1973).

[3] H. Heineken, 'Automorphism groups of torsion-free nilpotent groups of class 2', Symposia Mathematica 17 (1976), 235-250.

[4] D. J. S. Robinson, 'Outer automorphisms of torsion-free nilpotent groups', unpublished.

[5] U. Stammbach, Homology in group theory (Lecture Notes in Math., Vol. 359, Springer-Verlag, Berlin, 1972)

[6] R. B. Warfield, Nilpotent groups, (Lecture Notes in Math., Vol. 513, Springer-Verlag, Berlin, 1976).

[7] A. E. Zalesskii, 'An example of a torsion-free nilpotent group having no outer automorphisms', Mat. Zametki 11 (1972), 21-26; Math. Notes 11 (1972), 16-19.

\section{Science Division}

University of Wisconsin-Parkside

Kenosha, Wisconsin 53141

U.S.A. 